Running head: Culture and Diversity in Falls Prevention

\title{
The Role of Culture and Diversity in the Prevention of Falls among Older Chinese People*
}

\author{
Khim Horton \\ Faculty of Health and Medical Sciences, University of Surrey
}

Angela Dickinson

Centre for Research in Primary and Community Care, University of Hertfordshire

\section{RÉSUMÉ}

\begin{abstract}
This grounded-theory study explored the perceptions of Chinese older people, living in England, on falls and fear of falling, and identified facilitators and barriers to falls prevention interventions. With a sample of 30 Chinese older people, we conducted two focus groups and 10 in-depth interviews in Mandarin or Cantonese. Interview transcripts, back translated, were analyzed using N6. Constant comparative analysis highlighted a range of health-seeking behaviors after a fall: Chinese older people were reluctant to use formal health services; talking about falls was avoided; older people hid falls from their adult children to avoid worrying them; and fatalistic views about falls and poor knowledge about availability and content of interventions were prevalent. Cost of interventions was important. Chinese older adults valued their independence, and cultural intergenerational relations had an impact on taking action to prevent falls. Cultural diversity affects older adults' acceptance of falls prevention interventions.
\end{abstract}

- Ethics approval was gained from the Eastern Multi-Centre Research Ethics Committee (MREC) (05/mre05/11), and Research Governance approval from the relevant acute National Health Service Trusts and Primary Care Trusts. 
Manuscript received: / manuscrit reçu : 26/02/10

Manuscript accepted: / manuscrit accepté : 20/12/10

\section{Mots clés:}

Keywords: grounded theory, Chinese older people, falls, culture and diversity, prevention of falls

Correspondence and requests for offprints should be sent to / La correspondance et les demandes de tirés-à-part doivent être adressées à:

Khim Horton, Ph.D.

Division of Health and Social Care

Faculty of Health and Medical Sciences

University of Surrey

Guildford, Surrey GU2 7TE, United Kingdom

(k.horton@surrey.ac.uk)

This is an independent report commissioned and funded by the Policy Research Programme in the Department of Health. The views expressed are not necessarily those of the Department

\section{Introduction}

Falls among older people present a global problem. In the United Kingdom, about a third of older people aged 65 and older living in the community fall each year (Yardley, Donovan-Hall, Francis, \& Todd, 2006). Falls are the leading cause of serious accidental injury (resulting in admission to hospital for four or more days) among people aged 65 and older (Cryer, 2001). Older people are at particular risk of falls and fall-related injuries due to the physiological changes of aging - such as postural instability, gait disturbances, diminished muscle strength, poor vision, cognitive impairment, and taking multiple medications - as well as environmental hazards (Cryer, 2001; Dickinson et al., 2009; Lilley, Arie, \& Chilvers, 1995; Parker, Twemlow, 
\& Pryor, 1996). Apart from the injury sustained in a fall, a number of other potential consequences have been identified. These include (a) psychological problems, such as a fear of falling that results in restriction of activity; (b) loss of mobility; (c) an increase in dependency and disability; (d) hypothermia; (e) pressure-related injuries; and (f) infections (Department of Health [DH], 2001). Despite considerable research into falls, little is known about how falls affect older people from ethnic minority groups.

The prevention and management of falls in older people has become a key public health priority for governments (e.g. DH, 2001). In the United Kingdom, the National Institute of Clinical Excellence (NICE) has attempted to address the human and economic costs associated with falls and fractures through the publication of a national guideline on assessment and prevention (NICE, 2004). However, an examination of the literature on the role and influence of culture and diversity in falls prevention and intervention suggests that there has been little consideration of its potential impact. Increasing awareness of the need to address the cost and consequences of falling by local government, and by the United Kingdom and international agencies has led to strategic policies and health and social care initiatives on falls prevention and interventions (Roe et al., 2009). Recent publications by the United Kingdom Department of Health have focused on exercise training to prevent falls (Skelton \& Dinan, 2009), on development of joint strategic needs assessments (DH, 2009a), and on effective interventions in health and social care (DH, 2009b).

Ethnicity may affect attitudes towards and participation in falls-prevention strategies, but currently we have little understanding of this factor (Horton, 2002). A recent systematic review by Bunn, Barnet-Page, McInnes, Dickinson, and Horton (2008) noted that of the studies in English-speaking countries, only a few examined the health promotion needs of non-Englishspeaking groups (Aminzadeh \& Edwards, 2000; Edwards, Lockett, Aminzadeh, \& Nair, 2003; Health Education Board for Scotland, 2003). One recent ethnographic study by Horne, Speed, 
Skelton, \& Todd (2009) found that South Asian older people were more likely to have fatalistic understandings of falls that the study authors felt could affect their willingness to make changes to their behavior in response to health promotion messages. The authors found that falls prevention has a low priority among both Caucasians and South Asian older people in the United Kingdom.

Todd, Ballinger, and Whitehead (2007) reviewed the state of knowledge about falls and falls prevention among older people and considered how socio-demographic variables (such as age, gender, ethnicity, and socio-economic status) relate to the incidence of falls. In their review, ethnicity was referred to as "a multi-faceted quality that refers to the group to which people belong, and/or are perceived to belong, as a result of certain shared characteristics, including geographical and ancestral origins, but particularly cultural traditions and languages" (pp. 4-5). They noted that the relationship between falls and ethnicity remains wide open for research. They argued that ethnicity (unlike race) is not a simple biological concept but relates to a whole range of cultural practices (including religion), attitudes, beliefs, behaviors, and social structures. It is also associated with poverty, material deprivation, lack of access to resources, and power. Their review noted that the terms race and ethnicity were used interchangeably, especially in the U.S. literature, and they concluded that not only is it not possible to synthesize data relating to sociodemographic variables in any meaningful way but that more robust evidence is needed.

Further evidence suggests that there are cultural differences in understandings of health. Helman (2001), for example, argued that supernatural explanations for ill-health are more prevalent in non-Westernized individuals, whereas Westerners are more likely to explain illhealth as being related to factors within the individual or in the natural environment. Thus, an exploration of people's perceptions and experiences of falls would provide further insight into their health beliefs. From a sociological perspective, Wight (1999) argued that one aspect of modern culture is the reduced role of fate particularly in non-Western cultures, in which people 
would typically consider fate to play an important role. Some cultural theories posit that risk perception and behavior stem from the individual's "shared ways of interpreting the world" (Wight, 1999, p. 736). Wight further claimed that through cultural factors, an individual's behavior is, more often than not, shaped more by shared ways of interpreting the world than by the individual's cognition. Referring to the "ground" and "grid" theory of Douglas and Wildavsky (1982), Wight (1999) explained how an individual's perception of risk is sometimes linked to social organization, as different cultures are conceptualized with a "group" axis (i.e., the strength of the cultural group's boundary with the outside world) and a "grid" axis (i.e., the degree of social distinction within the cultural group). Wight highlights the need to view an individual as a "dynamic meeting point of many cultures" and cautions against interpreting their culture in a simplistic way.

This idea is closely related to diversity as individuals have unique characteristics, talents, and challenges reflected in the places they live, their socio-economic circumstances, ethnic heritage, religion, language, and abilities. Where health beliefs are concerned, the literature on culture draws our attention to the diverse expressions of the way people define and categorize health and illness. However, we could argue that this explanatory model for illness can be limiting: although falls are not a disease or illness, falls are associated with old age and frailty.

The Chinese population in the United Kingdom is the fifth largest ethnic group with four per cent of this population group aged 65 and older (Office for National Statistics, 2001). In common with other ethnic groups, Chinese older people are vulnerable due to language difficulties, racism, and reduced physical health; however, the circumstances of different groups may require different approaches to health care and the circumstances need to be understood in order for health professionals to deliver effective care (Butt \& O’Neil, 2004). In addition, Chinese older people are described as a community that is particularly invisible, socially and politically (Yu, 2000), with poor uptake of health and social services (Chau \& Yu, 2000). 
Chinese older people have been found to hold fatalistic beliefs in that falls are regarded as unavoidable and out of their control. In a Shanghai-based study by Xia, Jiang, Niu, Tang, and Xia (2009), a population-based community trial aimed to evaluate the effectiveness of an 18-month multi-faceted intervention to reduce the incidence of falls in the community for those older than age 60. Four residential communities were randomized as either a multi-faceted intervention or a control group. The researchers found a reduction in falls incidence in the intervention group. This study highlights the importance of "the community" as a focus in which there was a sense of relatedness to other members of the group. This suggests that identity and its cultural influence may play a role in how older people participate in falls interventions.

Globally, other studies also provide some evidence about Chinese older people. For example, using a qualitative approach, Yang, Haralambous, Angus, and Hill (2008) explored Chinese Australian older people's need for information in falls and falls prevention, and reported that falls were considered an important health issue. The authors noted the cultural belief that "If I have a fall, my children will have to look after me, but they are all busy with their work and family", which might explain why the study subjects reported a low awareness and utilization of other health services and professionals in falls prevention. The Chinese older people in this study believed that Chinese herbal medicine might be more effective in treating injuries caused by falls. Such behavior supports the findings of other studies (e.g., Kwok \& Sullivan, 2007, which found that Chinese Australian women's health-seeking behaviors meant that they would not see a medical practitioner until they felt sick or had a specific problem). This might explain why, in the United Kingdom, the percentage of Chinese people defining illness in themselves has been lower than in other groups (ONS, 2004).

Currently, uptake rates of community-based falls prevention interventions are very low, averaging about 50 per cent (Robertson, Devlin, \& Gardner, 2001), but can be as low as 10 per 
cent (Day, Fildes, \& Gordon, 2002). What is unclear is the uptake of such interventions or engagement in falls prevention activities by ethnic groups such as Chinese older people.

This article presents the findings from Chinese Older People who participated in a wider study involving Causacians, Asians and Chinese older people ${ }^{1}$. The study aimed first to explore the perceptions, attitudes, and beliefs of Chinese older people who had experienced a range of falls injury prevention programs, and, second, to identify the facilitators and barriers to their participation in those programs. For this article, only data from Chinese older people is discussed in order to explore the impact of culture on falls prevention and intervention.

\section{Methods}

We used a grounded-theory approach to develop an understanding of the barriers and facilitators to Chinese older people's accepting and adhering to falls prevention interventions. Grounded theory was strongly influenced by symbolic interactionism, an approach that respects a person's subjective interpretation of social experience as a source of knowledge. In grounded theory, participants are viewed as experts about their own experience.

We collected data that were mainly qualitative. Qualitative methods seek to understand human experiences, perceptions, motivation, and behavior, and are therefore appropriate for gaining an in-depth understanding of people's engagement with health care services (Lehoux, Poland, \& Daudelin, 2006).

Quantitative data was collected by means of a demographic pro forma, which included information such as age, sex, postal code, falls history, self-reported falls severity, and medication use, but it was used primarily to describe the demographic profile of the study sample. Participants were also asked to complete the Falls Efficacy Scale (Tinetti, Mendes de Leon, Doucette, \& Baker, 1994), which is a validated scale 
showing good test-retest reliability (Pearson's correlation=0.71) and construct validity (associations with avoiding activities, walking pace, anxiety and depression) (Tinetti et al., 1990). The scale also correlates with measures of balance and gait and has been found to predict future falls and declines in functional capacity (Yardley et al., 2005) and has been used to measure confidence in undertaking a range of physical and social activities such as washing, dressing, and walking around the neighborhood without falling. The FES is scored from 10 to 100 , with a score of 10 indicating 'extreme confidence' and a score of 100 indicating 'no confidence'. Since we began our data collection the FES has been superseded by the Falls Efficacy Scale-International (FES-I) which assesses a more difficult range of physical and social activities and is suitable for a range of languages and cultural contexts (Yardley et al., 2005).

\section{Sampling and Recruitment}

The Chinese substudy was based in one locality, in central London, England. In this study, we aimed to situate individual beliefs and practices within their socio-economic and cultural contexts, by collecting and analyzing data based on people's first-hand accounts, and attempting to retain the voices of the participants. Potential participants were informed that their involvement was voluntary and of the commitment required if they expressed an interest in taking part, and they were given at least 24 hours to consider their decision.

Participants were recruited from a Chinese voluntary organization that worked closely with local communities in central London. An important part of building trust with the Chinese groups of older people involved one of the researchers $(\mathrm{KH})$ spending time participating in classes with the group and taking part in the meal which was provided following the class. Interviews were conducted during November 2005 to March $2006^{2}$. In each focus group, participants were mixed, with fewer men than women. Nonetheless, this did not prevent either sex from making their 
views heard. Both focus groups were held in a private meeting room at a local center where tai chi classes took place. Data were collected after the tai chi sessions, for convenience as well as to ensure minimum disruption to people's lives.

Before the start of each focus group, time was provided for participants to ask further questions about the study, to sign consent forms, and to be given assistance, if required, in completing the demographic information we collected. We also explained the ground rules, which included items such as valuing each person's ideas, giving time for everyone to speak, trying not to talk "over" each other, and treating the discussion as confidential. A purposive sample of 30 Chinese older people $($ male $=9$, female $=21$, mean age 70.2$)$ who attended tai chi classes were recruited to take part in two focus groups with 10 people in each. An additional 10 people participated in one-to-one in-depth interviews. Most of the older people $(n=21)$ said they had experienced at least one fall in the previous two years. Additional information about participants, including marital status and mean FES score is included in Table 1.

[Insert Table 1 about here]

Each focus group discussion and one-on-one interview, both of which were taperecorded, lasted for between 60 to 75 minutes and was conducted in either Cantonese or Mandarin in which the first author $(\mathrm{KH})$ is fluent. All data collection tools were translated into Chinese. The interviews were fully transcribed to allow further detailed analysis. This ensured that cultural sensitivity and knowledge was applied to the data. Hakim (2000) had highlighted the problems of translation caused by national cultures and "taken-for granted assumptions", and pointed out that "most researchers agree that the aim is never a literal translation but functional equivalence of meaning" (Hakim, p. 207). 
Once our interviews were transcribed, ${ }^{3}$ they were anonymized by removing names and other identifying features and imported into N6 (QSR International Pty Ltd. Version 6, 2002), to enable data management and facilitate analysis of the data. Data were coded line-by-line, the codes identified were then grouped into themes. Both authors undertook an initial analysis of the data independently; this was followed by a discussion of the separate analyses during which time any differences in understanding and coding were resolved through discussion. Particular attention was paid to deviant cases that are exceptions within the data set (Green \& Thorogood, 2009).

\section{Findings}

The data we collected could be categorized into four general areas: (a) talking about and reporting of falls, (b) health beliefs about falls that included metaphysical explanations such as fatalism, (c) moral aspects concerning lifestyle, and (d) accessing falls prevention interventions.

\section{Talking About and Reporting Falls}

Chinese older people described a reluctance to talk about falls they had experienced. A number of reasons were given for this, including embarrassment, but also a preference to speak to friends about more-positive events in their lives. The following comment is from a study participant who described how falling in a public space was embarrassing, and how humor was used to overcome this.

I felt embarrassed too, especially when I once fell in front of my friend. We were out for a walk together and were busy talking. I must have tripped over something and missed my footing. I suddenly landed on my knees. One minute she was pointing to me about something, the next minute she found me on my knees. [laughter] I was very embarrassed. She was concerned but after a tea-break we had a laugh about it. She continued to tease me for a few weeks about that ... 
Among Chinese older people, there is also a sense that they prefer to speak of more-positive events with friends:

No, we talk about the weather, about other friends, any topic [about something that] is going [on], really: Christmas, New Year Sale, holidays, that sort of thing. Few occasions we hear of someone who went into hospital and then we talk about it. We try not to dwell [on] that sort of thing.

Chinese older people tried not to worry their adult children. This could be associated with concerns of dependency, vulnerability, and becoming a burden (Ballinger \& Payne, 2002).

... for a while I didn't want to tell my daughter. It was some months later only in general conversation that it cropped up. The look on her face told me that she was worried. She asked why I didn't tell her. I just told her that I must have forgotten ... I don't tell my family much about this type of thing. Why worry them. So long as I can still walk, do my own things - I don't want to worry them.

For Chinese older people, there was also a sense of loyalty to family, through taking care of themselves and therefore not to create worry (Chen, 2001).

\section{Older People's Health Beliefs about Falls}

Health beliefs about falls, and their causes and consequences, affect the way that information is interpreted and its impact on subsequent behavior, including the health-seeking behavior of older people. Our study revealed a number of popular lay beliefs about the causes of falls, which could have a consequence on the uptake and participation in falls prevention activities. Often reasons were associated with the aging body. 
I think everybody can fall but when an old person falls, it seems to be worse. Maybe it's because they are frail and weak already. From my experience, it took me a while to recover, so I see that as only one of the problems of growing old.

Chinese older people felt very strongly that they should wear shoes and clothing that was "appropriate" for their age.

Only that day, on the bus, this old lady, she must be older than me, and there she was, with all the makeup and all dressed up. She even had high heels on. If she falls, I think to myself, you've only got yourself to blame. Wearing heels like what she had on, she's only asking for trouble. I just wear these trainers that my son bought me. It's comfortable, ideal for tai chi and walking about. Why bother with those fanciful footwear ....

The following comment from one of the study's Chinese participants obviously sees falls as something that is preventable, which she is taking action to avoid through tai chi exercise:

I haven't had a fall, and that's why I want to continue doing tai chi because I think doing exercise like this helps me. I think it makes me stronger. My muscles - my leg muscles - are stronger, I think.

For Chinese older people, eating and food played an important part in staying healthy and well. Eating traditional Chinese food was very important to maintaining health, and there was reference to the hot/cold system of foods, as well as the need to eat the traditional Chinese staple of rice as well as using Chinese herbs as indicated here by two participants:

Of course, you can tell whether your body is in good health. You don't need the newspaper to tell you. If I don't feel well, I know it. I will quickly boil some herbal tea. Sometimes, the weather is no good; you eat too much heaty food. You have to drink tea... 
We have been brought up eating rice, of course we miss them. I don't really like trying Western food. Not even milk. I don’t like it.

\section{Metaphysical/Supernatural Explanations of Falls}

Chinese older people in this study described a lack of exposure to public health messages and activity relating to the causes and prevention of falls. This appeared to leave people to draw, in the main, on their own lay knowledge and ideas for explanations and responses in terms of action.

In the absence of formal or expert knowledge about falls, including their causes and prevention, many older people drew on ideas in the metaphysical and supernatural realm of beliefs. These beliefs included fatalistic ideas by the Chinese older people we spoke to: they perceived it to be luck, rather than divine intervention, as to whether they fell or not, and falls were frequently described as accidental or chance events. These ideas of unpredictability led to falls being considered, in the main, to be non-preventable.

Older people in the study used the term "luck" or "lucky" but this appears to be more a turn of phrase with little sense of their meaning that some "supernatural" force was at play. Some Chinese older people used the word luck in this manner: for example, "I was very lucky, very lucky that I didn't fall down those steps" [interview with Chinese older man].

Due to the Chinese older people's poor understanding of the English language, they could be seen to be culturally isolated and therefore more likely to be strongly influenced by their culturally influenced traditional views of illness. Chinese older people do not draw solely on notions of luck to explain falls; however, luck as a factor in the experience of falling was a particular feature of the interviews with the Chinese older people. 
Those people who had a strong sense of fatalism, who viewed falling as part of their destiny or punishment, were less likely to view falls as preventable or interventions as effective or worth participating in because they could do little or nothing to affect the outcome:

You can do something about it [fall] but I think it sometimes will happen no matter what...

Chinese older people recognized that luck and superstition were part of their culture:

Maybe we Chinese always talk about luck [laughs]. Luck is important. You're lucky if you win the lottery. You're unlucky if you fall [laughs]. But, seriously, it's not that easy to say if falling has to do with bad luck. It could be bad luck, maybe but I don't know. Some people are more superstitious than others.

Both having the fall and the outcome are attributed to good or bad luck. Finding the tai chi class was also attributed to luck:

My family is pleased that I have learned to do tai chi. They know I had wanted to do that but didn't know how to find out. I was very lucky that the pastor mentioned it.

However, fatalism was not a belief of all Chinese older people; other health beliefs were evident, illustrating the complexity of beliefs. Here, beliefs about the fall are located within the aging body, rushing, and carelessness.

But, if you think about it, it's got nothing to do with having luck or not. If your body caused you to fall, you fall. Nothing to do with luck or no luck. Nothing to do with fate either. I think when I had that fall, it had to do with my weaker leg. It gave way and I fell. It wasn't about whether I was lucky or not that my leg gave way... 
Sometimes, I think it's also got to do with not taking care, you're rushing here and there and not thinking that your body cannot be rushed.

\section{Moral Aspects of Lifestyle}

The following observations highlight the moral elements associated with this category. There was a feeling that people should do whatever they could to reduce falls; those who did not were viewed critically as being at fault. There was also a sense of critical self-judgment following a fall. Words such as "stupid" and "own fault" were frequently used to locate their beliefs about blame when describing falls experiences.

Falls could be prevented? ...I think there must be times when a person can stop it. It's like if you deliberately go out when it's icy and you don't wear proper shoes and you fall, then it's your own stupid fault.

And:

It was my own fault, not looking where I was going.

It would appear that their lifestyle risk reduction included a focus on keeping their body healthy as well as making efforts that are "directed towards maximizing one's health and minimizing one's burden on society", risks are direct consequences of what someone does or does not do and thus have moral elements (Kavanagh \& Broom, 1998, p. 438).

\section{Accessing Fall Prevention Interventions}

Chinese older people had great difficulty finding out information about falls and falls prevention interventions. Lack of knowledge about services was exacerbated by language 
difficulties but also literacy problems. Many of the participants were unable to read their own language, so translation of text-based information would not have been useful. The Chinese older people valued the tai chi classes they attended, particularly as the teacher spoke Mandarin and Cantonese.

It is good that we have it in Chinese. I will not attend if it's not in Chinese.

When asked about how they would prefer information about falls to be presented, suggestions included using visual media such as television or DVD presented in Chinese. Word of mouth was considered very important for transmitting information (e.g., through friends). Information requiring a computer to access it was felt to be of little use:

Oh computer, that's no good to me. Ask my grandchildren and they'll know but not me. Why would I want to know about computers at my age?

The older people enjoyed the social aspects and the community feel of the tai chi exercise groups, attending with and making friends. Many of the participants also stayed and ate the Chinese food offered after that class, and felt that this was important to them.

I make friends and I enjoy my food after the class. It's also a social thing; we catch up with news from others. It's just like a big family.

Chinese older people also stressed the importance that the intervention should be culturally appropriate. Tai chi was suitable due to its Chinese origins, but dance-based interventions were deemed totally unsuitable.

But I am not into that sort of thing. Too old now. What would my daughter think of me dancing? At my age, it's a laughing matter. My dead husband would have been shocked. 
Generally, the cost of an exercise program was not perceived to be a barrier to participation as long as this was "reasonable", although the participants valued the intervention's being subsidized in order to keep the cost low.

For me, I wouldn't mind paying the odd pound but I guess it can mount up if you come twice a week, every week in the month.

\section{Discussion}

It is clear from the findings that there are cultural impacts reflected in the responses of Chinese older people to both falls and falls prevention interventions. There are also similarities with responses from other cultures. For example, the reluctance to discuss falls supports Kingston (2000), who found that almost 80 per cent of older people who had recently fallen had not discussed the fall with either family or friends. Kingston proposed that falls marked "undesirable" status passages, where individuals attempt to keep evidence of their changing or deteriorating health status secret because of the fear of being unable to maintain their independent lives in the community and having to move into residential care. Likewise, the Chinese participants in our study preferred to talk about positive life events, and wished to protect their families from worrying about them.

Davison, Davey Smith, and Frankel (1991) argued that within the field of classical anthropology, cultural systems of explanation must address two issues: first, how and why misfortunes happen; and second, how and why it happened to this person at this particular time. They stated that in order to answer the former, people draw on physical and material processes; however, to explain the latter, people draw on ideas of a metaphysical or supernatural origin. The researchers stressed that these ideas are used in the absence of an "adequate aetiological 
hypothesis" (p. 14). In this study, metaphysical and supernatural ideologies, though diverse in origin, all had as a central feature the notion that there was nothing that could be done by the individual to prevent falls. This has major implications for falls prevention programs.

Our sample of Chinese older people drew heavily from the metaphysical domains of health beliefs to explain their experiences of falls. As Shearer and Davidhizar (2000) expressed it: “ ... for an individual who does not have the benefit of knowledge or experience, a positive result would seem unaccountable except by sheer luck" (p. 3). These ideas may be drawn on in the absence of other information. These health beliefs need to be understood and worked with by those health professionals promoting falls prevention activities; however, they should not be perceived to be a reason for non-referral or for neglecting prevention work in these population groups. Hence, we argue that prevention interventions need to be culturally appropriate if they are to be accepted (e.g., Chinese older people described how they would find dance-based activities inappropriate, but tai chi was acceptable because it originated in China).

Beliefs that outcomes are randomly determined by forces such as luck, fate, and chance, and thus that control lies beyond the ability of the individual, are described by psychologists as indicative of an "external locus of control" (Rotter, 1966). The internal and external loci of control (LOC) are at opposite ends of a continuum. Those at the internal LOC end of the continuum believe that outcomes are the direct result of the individual's behavior. Individuals with an external LOC are less likely to take responsibility for their actions and to make behavioral changes to improve their health.

The interviews with older Chinese participants illustrate how "lay theories may display a complex and thoughtful interest in the relationship between preventability and inevitability, an area sometimes glossed over by the confidence in control which pervades the ideology of modern Western medicine" (Davison et al., 1991, p. 4). The metaphysical was described in the quotes 
from study participants using terms influenced by older people's cultural and religious backgrounds. In our study, we saw how Chinese older people drew on the idea of "luck" to explain falls experiences. Interestingly, such fatalistic ideas were common in both older men and women, although we acknowledge that there were too few men in our sample group to make strong claims regarding gender differences.

A study by Gervais and Jovchelovitch (1998) of the health beliefs of Chinese people living in the United Kingdom reported that superstitious and extremely fatalistic beliefs were rare, and were found only in older Chinese people who were less educated and had immigrated from rural areas. That study did not include older people, relying on reporting from younger Chinese people. Thus the researchers did not have any direct evidence of the health beliefs of older Chinese people. In a different study, Horne et al. (2009) found a tendency for South Asians to hold fatalistic beliefs. Nonetheless, their findings showed that those who had fallen were more likely to acknowledge their risk of future falls and, when informed of the benefits of interventions such as exercise, were more likely to consider participating in a falls prevention program.

A number of studies of the health beliefs of Chinese people in other areas indicate a strong influence of "folk beliefs" in the understanding of Alzheimer's disease (Jones, Chow, \& Gatz, 2006) and disbelief in the effectiveness of oral health prevention and use of traditional remedies (Kwan \& Holmes, 1999). Low uptake of mammography, for example, by Chinese women was found to be influenced by beliefs such as fear of radiation and the procedure (Kwok, Cant, \& Sullivan, 2005). Chinese philosophies and religions have also been found to affect health beliefs (Chen, 2001).

Our study has some limitations. The findings from qualitative research are context-specific and caution is required in translating these findings to other areas/groups. The sample did not include older people with cognitive impairments, and therefore, further research is required to explore the requirements of this group of people. The grounded-theory approach we used is 
considered appropriate for the study, and although some time was spent with the groups being studied prior to recruitment and data collection, an ethnographic methodology could develop understanding further. More work where researchers spend time in community settings to enable them to be "accepted" by the minority community being investigated is required if researchers are to understand social meanings from the community's perspective. An ethnographic study with this minority group could provide a richer basis for us to understand the cultural context of falls and falling among older Chinese people.

Culturally appropriate falls prevention programs should take into consideration the specific issues of this ethnic group (e.g., to promote suitable preventive interventions such as tai chi exercise groups with instruction presented in their own languages). We would argue that exploring the cultural context from older people's perspectives is important if we are to begin to understand more about why some people might be willing to participate in falls prevention interventions, such as tai chi and other balance exercise classes, and when developing services for older people from other ethnic groups. The challenges of recruiting older people from minority groups to falls prevention programs must be addressed by both policy makers and health and social care professionals.

\section{Conclusion}

The responses of Chinese older people to both falls and falls prevention interventions are varied and complex. The Chinese older people in our study were reluctant to use formal health services, avoided talking about falls, and hid falls from their adult children to avoid worrying them. Fatalistic views about falls and poor knowledge about availability and content of interventions were prevalent. Chinese older adults valued their independence, and cultural intergenerational relations had an impact on taking action to prevent falls. 
There are no simplistic recipes to follow to increase Chinese older people's levels of uptake. The evidence from this study indicates that lay health beliefs about falls impacts older people's response to both the fall experience and the available interventions. Although this study presented data only on people from one specific ethnic group, it is clear that there are key areas that must be addressed in order to provide effective services to this and to other ethnic groups of older people. As many older people from ethnic minority groups may not be able to read or speak English, information about falls and falls prevention interventions should be published and disseminated in a range of languages. Instructors and facilitators also need to speak the language of the target groups.

\section{Notes}

1. We undertook a qualitative study with older people who had taken part in, or declined to participate in fall prevention interventions using semi-structured interviews ( $\mathrm{n}=65)$, and 17 focus groups ( $\mathrm{n}=122)$ with older people (including 32 Asian and 30 Chinese older people). This took place in community settings in four geographical areas of the South of England. This article reports only data from the Chinese participants.

2. The topic areas for discussion in the focus groups were developed by the research team following the systematic literature review (Phase 1 of the study, Bunn et al., 2008 with the additional input of the project steering group. For one-to-one interviews, the interviewer introduced the topic and guided the discussion by asking specific questions. Semi-structured interviews have a flexible structure made up from a series of open-ended questions around the subject which was being researched. For a copy of the interview guide, please e-mail the corresponding author directly. 
3. All data were transcribed by KH. The information leaflet was translated into Chinese.To ensure that the translation was accurate, back translation was undertaken by a Chinese-speaking member of the Chinese Woking Association who confirmed the credibility of the translation.

\section{References}

Aminzadeh, F., \& Edwards, N. (2000). Factors associated with cane use among community dwelling older adults. Public Health Nursing, 17(6), 474-483.

Ballinger, C., \& Payne, S. (2002). The construction of risk of falling among and by older people. Ageing and Society, 22, 305-324.

Bunn, F., Barnet-Page, E., McInnes, E., Dickinson, A., \& Horton, K. (2008). A systematic review of older people's perceptions of facilitators and barriers to participation in falls-prevention interventions. Ageing and Society, 28, 449-472.

Butt, J., \& O’Neil, A. (2004). 'Let's move on' Black and minority ethnic older people's views on research findings. York, UK: Joseph Rowntree Foundation.

Chau, R., \& Yu, S. (2000). From double attachment to double detachment: Chinese older people in Britain. In T. Maltby, A. Warnes, \& L. Warren (Eds.), Care services for later life:

Transformations and critique. London, UK: Jessica Kingsley.

Chen, Y. (2001). Chinese values, health and nursing. Journal of Advanced Nursing, 36(2), 270273.

Cryer, C. (2001). What works to prevent accidental injury among older people? Report to the Health Development Agency, London. Centre for Health Services Studies, University of Kent, UK. 
Davison, C., Davey Smith, G., \& Frankel, S. (1991). Lay epidemiology and the prevention paradox: The implications of coronary candidacy for health education. Sociology of Health and Illness, 12, 1-19.

Day, L., Fildes, B., \& Gordon, I. (2002). Randomised Factorial trial of falls prevention among older people living in their own homes. British Medical Journal, 325, 128-131.

Department of Health (DH). (2001). National Service Framework for older people. London, UK: Department of Health.

Department of Health (DH). (2009a). Falls and fractures: Developing a local joint strategic needs assessment. London, UK: Department of Health.

Department of Health (DH). (2009b). Falls and fractures: Effective interventions in health and social care. London, UK: Department of Health.

Dickinson, A., Machen, I., Cove, J., Horton, K., Jain, D., \& Maddox, T. (2009). Phase 2:

Facilitators and barriers to older people accepting and adhering to interventions to reduce falling and fractures: A qualitative exploration. Final Report to the Department of Health, London, UK.

Douglas, M., \& Wildavsky, A. (1982). Risk and culture: An essay on the selection of technical and environmental dangers. Berkeley, CA: University of California Press.

Edwards, N., Lockett, D., Aminzadeh, F., \& Nair, R. (2003). Predictors of bath grab-bar use among community-living older adults. Canadian Journal on Aging, 22(2), 217-227.

Gervais, M.C., \& Jovchelovitch, S. (1998). The health beliefs of the Chinese community in England: A qualitative research study. London, UK: Health Education Authority.

Green, J. \& Thorogood, N. (2009) Qualitative methods for health research. London, UK: Sage. 
Hakim, C. (2000). Research Design successful designs for social and economic research.

London, UK: Routledge.

Health Education Board for Scotland. (2003). The construction of the risks of falling in older people: Lay and professional perspectives. Edinburgh, UK: Health Education Board for Scotland.

Helman, C.G. (2001). Culture, health and illness. London, UK: Arnold.

Horne, M., Speed, S., Skelton, D., \& Todd, C. (2009). What do community-dwelling Caucasian and South Asian 60-70 year olds think about exercise for fall prevention? Age and Ageing, 38, $68-73$.

Horton, K. (2002). Gender and falls: Older people and key family members' perspectives.

Unpublished PhD thesis, Department of Sociology, University of Surrey, Guildford, Surrey, UK.

Jones, R.S., Chow, T.W., \& Gatz, M. (2006). Asian American's and Alzheimer's disease:

Assimilation, culture and beliefs. Journal of Aging Studies, 20, 11-25.

Kavanagh, A.M., \& Broom, D.H. (1998). Embodied risk: My body, myself? Social Sciences and Medicine, 46(3), 437-444: 438.

Kingston, P. (2000). Falls in later life: Status passage and preferred identities as a new orientation. Health, 4(2), 216-233.

Kwan, S.Y.L., \& Holmes, M.A.M. (1999). An exploration of oral health beliefs and attitudes of Chinese in West Yorkshire: A qualitative investigation. Health Education Research, 14(4), 453460.

Kwok, C., Cant, R., \& Sullivan, G. (2005). Factors associated with mammographic decisions of Chinese-Australian women. Health Education Research, 20(6), 739-747.

Kwok, C., \& Sullivan, G. (2007). Health seeking behaviours among Chinese-Australian women: Implications for health promotion programmes. Health, 11(3), 401-415. 
Lehoux, P., Poland, B., \& Daudelin, G. (2006). Focus group research and 'the patient's view'. Social Science and Medicine, 63(8), 2091-2104.

Lilley, J.M., Arie, T., \& Chilvers, C.E.D. (1995). Accidents involving older people: A review of the literature. Age and Ageing, 24, 346-365.

National Institute for Clinical Excellence (NICE). (2004). Falls: The assessment and prevention offalls in older people. Clinical Guideline 21 November 2004. London, UK: NICE.

Office for National Statistics (ONS). (2001). Focus on older people \& ethnic minorities.

Retrieved October, 2010, from http://www.statistics.gov.uk/focuson/ethnicity

Office for National Statistics (ONS). (2004). Health expectancies at birth and age 65 in the UK. London, UK: ONS.

Parker, M.J., Twemlow, T., \& Pryor, G. (1996). Environmental hazards and hip fractures. Age and Ageing, 25, 322-325.

Robertson, M.C., Devlin, N., \& Gardner, M.M. (2001). Effectiveness and economic evaluation of a nurse delivered home exercise programme to prevent falls: A randomised controlled trial. British Medical Journal, 322, 697-701.

Roe, B., Howell, F., Riniotis, K., Beech, R., Crome, P., \& Ong, B.N. (2009). Older people and falls: Health status, quality of life, lifestyle, care networks, prevention and views on service use following a recent fall. Journal of Clinical Nursing, 18, 2261-2272.

Rotter, J.B. (1966). Generalized expectancies for internal versus external control of reinforcement, Psychological Monographs, 80, (1, Whole No. 609).

Shearer, R., \& Davidhizar, R. (2000). Luck, what the nurse should know about it and how it affects nursing situations. International Journal of Nursing Practice, 6(1), 2-6. 
Skelton, D.A., \& Dinan, S.M. (2009). Falls and factures: Exercise training to prevent falls. London, UK: Department of Health.Tinetti, M.E., Richman, D., \& Powell, L. (1990) Falls efficacy as a measure of fear of falling. Journal of Gerontology, 45, P239-P243.

Tinetti, M.E., Mendes de Leon, C.F., Doucette, J.T., \& Baker, D.I. (1994). Fear of falling and fall-related efficacy in relationship to functioning among community-living elders. Journal of Gerontology, 49, M140-M147.

Todd, C., Ballinger, C., \& Whitehead, S. (2007). Reviews of socio-demographic factors related to falls and environmental interventions to prevent falls amongst older people living in the community. Copenhagen, Denmark: World Health Organization.Regional Office for Europe.

Wight, D. (1999). Cultural factors in young heterosexual men's perception of HIV risk. Sociology of Health and Illness, 21(6), 735-758.

Xia, Q.H., Jiang, J., Niu, J., Tang, C.X., \& Xia, Z.L. (2009). Effectiveness of a community-based multifaceted fall-prevention intervention in active and independent older Chinese adults. Injury Prevention, 15, 248-251.

Yang, X.J., Haralambous, B., Angus, J., \& Hill, K. (2008). Older Chinese Australians' understanding of falls and fall prevention: Exploring their needs for information. Australian Journal of Primary Health, 14(1), 36-42.

Yardley, L., Beyer, N., Hauer, K., Kempen, G., Piot-Ziegler, C., \& Todd, C. (2005). Development and initial validation of the Falls Efficacy Scale-International (FES-I). Age and Ageing, 34(6), 614-619.

Yardley, L., Donovan-Hall, M., Francis, K., \& Todd, C. (2006). Older people's views of advice about falls prevention: A qualitative study. Health Education Research, 21(4), 508-517. 
Yu, W.K. (2000). Chinese older people: A need for social inclusion in two communities. Bristol, UK: The Policy Press and the Joseph Rowntree Foundation. 
Table 1: Characteristics of the participants in the study of Chinese older people

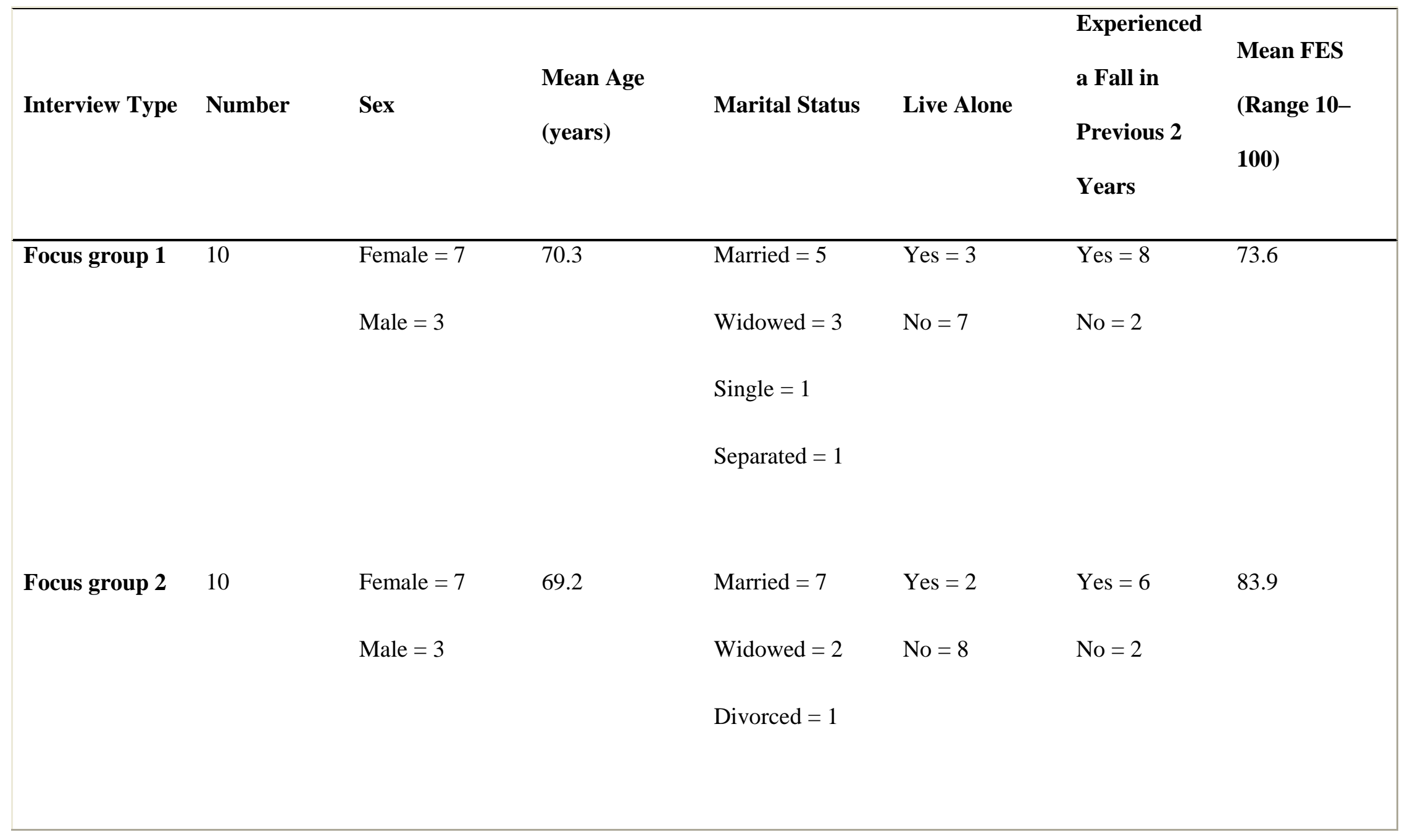




$\begin{array}{cccccc}\text { Interview } 10 & \text { Female }=7 & 71.1 & \text { Married }=4 & \text { Yes }=1 & \text { Yes }=7 \\ & \text { Male }=3 & \text { Widowed }=6 & \text { No }=9 & \text { No }=3 & 38.3 \\ \end{array}$

FES = Falls Efficacy Scale 